\title{
Verzeichnis der Autorinnen und Autoren
}

Angelika Beranek | ist Professorin und Studiendekanin an der Hochschule München mit dem Schwerpunkt Medienbildung. Sie beschäftigt sich mit den Auswirkungen der Digitalisierung auf die Theorie und Praxis der Sozialen Arbeit. Zuletzt hat sie als Mitherausgeberin den Sammelband „Big Data, Facebook, Twitter und Co. und Soziale Arbeit“ herausgebracht. [@a_beranek]

Juliane Besters-Dilger $\mid$ ist Prorektorin für Studium und Lehre an der Albert-Ludwigs-Universität Freiburg und unter anderem zuständig für Lehrentwicklung und Hochschuldidaktik. Sie ist ferner verantwortlich für Exzellenz und Qualitätsmanagement in der Lehre.

Marko Demantowsky | ist Professor für Neuere/Neueste Geschichte und ihre Didaktik an der Pädagogischen Hochschule FHNW (Leiter der Professur für die Didaktik der Gesellschaftswissenschaften und ihre Disziplinen) und Mitglied des Instituts für Bildungswissenschaften der Universität Basel. \#DKMMZ19-Team. [@mdemantowsky]

Thomas Grob | ist Vizerektor Lehre an der Universität Basel. Er ist seit 2009 Professor für Slawische und Allgemeine Literaturwissenschaft an der Universität Basel. Er ist unter anderem für die Hochschuldidaktik zuständig.

Sarah Genner | ist Medienwissenschaftlerin und Dozentin an verschiedenen Schweizer Hochschulen. Sie war Gastforscherin am Berkman Klein Center for Internet and Society der Harvard University. Ihre Dissertation zum mobilen Internet wurde mit dem Mercator-Award der Universität Zürich ausgezeichnet. [@sgenner]

Jürgen Hermes | ist Geschäftsführer des Instituts für Digital Humanities an der Universität zu Köln und dort zuständig für die Konzeption und Betreuung der Studiengänge, die Studierende ausbilden, digitale Methoden und Werkzeugen zur Anwendung auf geisteswissenschaftliche Daten und Fragestellungen zu entwickeln. Schlechter Visionär, besserer Verbinder. Bloggt TEXperimenTales. [@spinfocl] 
Ute Kalender | war Postdoc in den Kultur- und Genderwissenschaften an der Humboldt-Universität Berlin und assoziiert im Graduiertenkolleg "Geschlecht als Wissenskategorie“. Ihr letztes ethnografisches Forschungsprojekt untersuchte affektive Arbeit in Daten-Zentren der derzeit größten deutschen Big-Data-Gesundheitsstudie. In ihrem aktuellen Forschungsprojekt untersucht sie zusammen mit Aljoscha Weskott Verständnisse von Digitalität und digitaler Geschlechtergerechtigkeit in feministischen sozialen Bewegungen. Sie ist wissenschaftliche Mitarbeiterin in der Sozialmedizin der Charité. [@kalendeu]

Christoph Kappes | befasst sich seit über 20 Jahren mit der Digitalisierung, immer schon mit wirtschaftlichen und technisch-konzeptionellen, seit einigen Jahren auch mit gesellschaftlichen Fragen. Er war Gründer und Geschäftsführer der heutigen Pixelpark Agentur Hamburg GmbH. Seit 2008 ist er Geschäftsführer im Beratungsgeschäft Fructus GmbH. [@ChristophKappes]

Björn Klein | ist Historiker und war Lehrbeauftrager an der Universität zu Köln. Er wurde an der Georg-August-Universität Göttingen promoviert und war Mitglied und Sprecher am dortigen Graduiertenkolleg „Dynamiken von Raum und Geschlecht“. Er arbeitet zu Trans- und Intersektionalität, Nordamerikanischer Geschichte, Geschlechtergeschichte und digitaler Bildung. Seit 2018 ist er wissenschaftlicher Mitarbeiter an der Pädagogischen Hochschule FHNW. [@john_doneson]

Gerhard Lauer | ist Literaturwissenschaftler und Professor für Digital Humanities an der Universität Basel. Er ist Mitglied der Akademie der Wissenschaften zu Göttingen, Mitgründer des Journal of Literary Theory und Mitherausgeber des Zeitschrift Scientific Study of Literature. \#DKMMZ19-Team. [@GerhardLauer]

Dejan Mihajlović $\mid$ setzt sich mit der Bildung von morgen auseinander und möchte sie heute schon denken. Er ist Chemie-, Geschichts-, Matheund Ethiklehrer; Multiplikator der „Fortbildungsoffensive Digitalisierung" an allgemeinbindenden und beruflichen Schulen beim Kultusministerium Baden-Württemberg und netzpolitisch bei „D64 - Zentrum für digitalen Fortschritt“ zuhause. [@DejanFreiburg]

Christian Montag | ist Heisenberg-Professor für Molekulare Psychologie in Ulm sowie Professor an der University of Electronic Science and 
Technology of China in Chengdu, China. Er forscht unter anderem zu Psychoinformatik und hier insbesondere zum Einfluss von Internet, Mobiltelefonen, Computerspielen auf Emotionalität, Persönlichkeit und Gesellschaft. [@ChrisMontag77]

Kathrin Passig | ist Journalistin und Sachbuchautorin, zuletzt von „Handbuch für Zeitreisende: Von den Dinosauriern bis zum Fall der Mauer" (Rowohlt Berlin, zusammen mit Aleks Scholz). Gemeinsam mit einigen hundert Autorinnen und Autoren berichtet sie im Blog „Techniktagebuch“ (https://techniktagebuch.tumblr.com/) über Alltagstechnik und deren Veränderungen. [@kathrinpassig]

Robin Schmidt | ist seit 2001 Leiter der Forschungsstelle Kulturimpuls und arbeitet derzeit am Projekt „Menschlichkeit der Digitalmoderne“. Seit 2016 ist er auch wissenschaftlicher Mitarbeiter an der Pädagogischen Hochschule der FHNW mit einem Forschungsprojekt zu Lehren und Lernen im Digitalen Wandel. \#DKMMZ19-Team. [@_robinschmidt]

Monika Stiller Thoms | ist Lehrerin für Deutsch und setzt sich schwerpunktmässig mit digitalen Medien und Theaterpädagogik in der Schule auseinander. Sie sammelt auf ihrem Blog „Digitale Chancen“ Methoden und Materialien zu digitalen Themen im Deutschunterricht der Sekundarstufe II. [@ichbinstiller]

Philippe Wampfler $\mid$ ist Lehrer, Fachdidaktiker, Kulturwissenschaftler und Experte für Lernen mit Neuen Medien. Sein zuletzt erschienenes Buch heißt „Schwimmen lernen im digitalen Chaos“. Der Fokus seiner Arbeit liegt auf den Entwicklungsmöglichkeiten gymnasialer Bildung unter den Bedingungen der Digitalisierung. [@phwampfler]

Marina Weisband $\mid$ ist Autorin, Künstlerin, Projektleiterin, Psychologin und Beraterin zu netzpolitischen Fragen. Ihr 2013 erschienenes Buch trägt den Titel ,Wir nennen es Politik, Ideen für eine zeitgemäße Demokratie". Sie hat sich in unterschiedlichsten Kontexten mit (Schul-) Bildung auseinandergesetzt, unter anderem leitete sie 2014 das von der Bundeszentrale für politische Bildung geförderte Projekt „Aula - Schule gemeinsam gestalten“. [@afelia]

Bert Theodor te Wildt $\mid$ ist Facharzt für Psychiatrie und Psychotherapie. Er ist Chefarzt der Psychosomatischen Klinik Dießen. Zuvor leitete er die 
Ambulanz der LWL-Universitätsklinik für Psychosomatische Medizin und Psychotherapie an der Ruhr-Universität Bochum, wo er im Oktober 2012 die Medienambulanz mit der Sprechstunde für Menschen mit Internet- und Computerspielabhängigkeit begründete. Sein jüngstes Buch trägt den Titel „Digital Junkies - Internetabhängigkeit und ihre Folgen für uns und unsere Kinder“. \#DKMMZ19-Team. [@berttewildt] 